\title{
Evaluating the Efficiency of Polyhedral Mesh Elements in Solving the Problem of the Flow around Ship's Rudder
}

\author{
AMJD IBRAHEEM \\ Budapest University of Technology and Economics, department of Aeronautics, Naval and Railway Vehicles, \\ amjd.ibraheem@edu.bme.hu
}

Abstract: Meshing is one of the crucial key features to the success of CFD based simulations, this study is evaluating the efficiency of polyhedral elements in solving the problem of the flow around ship rudder, using a Reynolds-averaged Navier-Stokes turbulence model (SST $k$ - $\omega$ ), and compares it to a tetrahedral based mesh, considering that polyhedral elements were neglected in the past due to difficulties in implementing them, this was solved by introducing a tool by ANSYS that converges tetrahedral elements to polyhedral element, and integrating it into FLUENT software, the model was validated by comparing it with previously validated model which used the full version of ANSYS, this study was concluded using the academic version, but still it was able to produce satisfying results in predicting the lift and the drag coefficient, the pressure around the rudder surface, the velocity and the turbulent kinetic energy, finally the mesh quality was evaluated using the orthogonal quality criteria, the results showed the supremacy of the polyhedral elements in saving time and computational resources and improving mesh quality, and keeping high level of accuracy in predicting the results.

Keywords: CFD, Mesh, Rudder, Simulation.

\section{Introduction}

Solving the problem of the flow around ship rudder is considered one of the main applications of CFD as it saves cost and time compared to water tunnels (towing tank) traditional setups, and it proved its efficiency and ability to produce comparable and acceptable results. Lloyd's Register has used CFD considerably in analyzing the factors that affect the drag on different types of rudders (Whitworth, 2007), CFD is widely used also in universities known for its excellence in naval architecture and marine engineering as Delft University (Kim, 2011). Rudders were one of the main research topics which depended on CDF and the results were validated by comparing it to actual tunnel experiments, (Liu \& Hekkenberg, 3D RANS simulations of shallow water effects on rudder hydrodynamic characteristics, 2016) studied the shallow water effects on NACA 0020 rudder hydrodynamics using 3D RANS simulations and their results were validated through comparing it with wind tunnel results.

The key to a successful CFD based simulation is the mesh generation (Sadrehaghighi, 2020), for a long time the usage of hexahedral elements was very popular as it showed great flexibility, the problem was that generating this kind of meshes was a complicated process that requires both time and experience, especially when dealing with complex geometries. Thus, hexahedral mesh generation continued to be 
difficult to perform and automate (Shepherd \& Johnson, 2008). On the other hand, tetrahedral elements overcome some of the hexahedral mesh disadvantages, as they are much easier to generate and consume less computational resources regardless of the geometry complexity, but one disadvantage is the high probability of numerical diffusions, the other is the need for very fine tetrahedral elements near the walls or the combined use of tetrahedral and prismatic elements. Finally, there are the polyhedral elements, which did not draw attention in the past because they were not integrated into popular CFD tools like ANSYS, Around 2010, generalized polyhedral elements started to gain attraction in many CFD applications and it was introduced to ANSYS FLUENT solver (ANSYS, 2018), the major advantage of polyhedral elements is that every element has a lot of neighboring elements, and this leads to a much better approximation of gradients, in addition to that polyhedral elements has less sensitivity towards stretching compared to tetrahedral elements, polyhedral elements can also achieve better accuracy compared to hexahedral elements because of the higher number of neighboring elements. Mass is exchanged over a higher number of faces, which reduces the numerical diffusion effects caused by nonperpendicular flows to any of the cell faces (Sosnowski, Krzywanski, \& Gnatowska, Polyhedral meshing as an innovative approach to computational domain discretization of a cyclone in a fluidized bed CLC unit , 2017), it was noticed in some cases of complex geometries that polyhedral meshes can resource hungry. For the overall calculation time, the calculation time for the polyhedral meshes is three to five times faster than the calculation time of tetrahedral meshes, depending on each case. (Sosnowski, Krzywanski, \& Gnatowska, Polyhedral meshing as an innovative approach to computational domain discretization of a cyclone in a fluidized bed CLC unit, 2016)

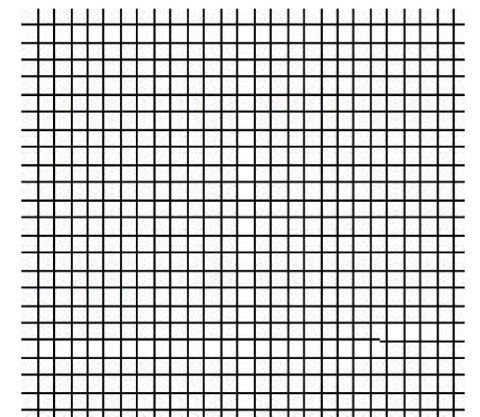

(a)

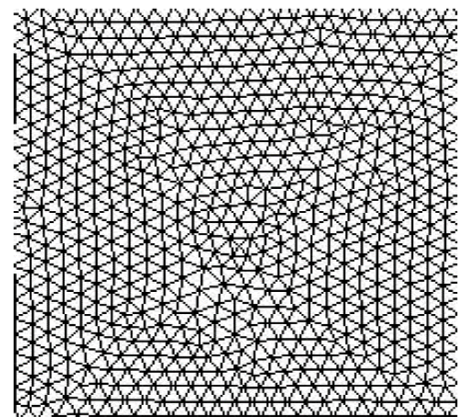

(b)

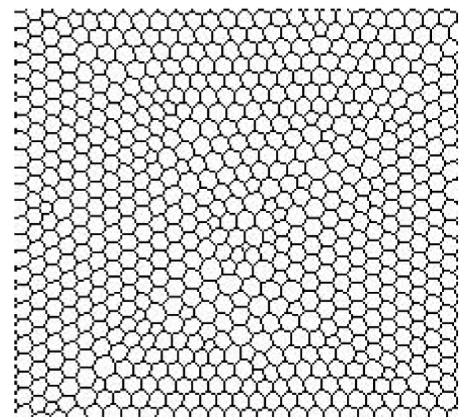

(c)

Figure 1. Three mesh types (a) hexahedral; (b) tetrahedral; (c) polyhedral (Michalcová \& Kotrasova, 2020)

Meshing rudder shaped objects is very widely presented in the literature, different types of meshes were applied to solve different problems, in 2014 Van Nguyen and Ikeda (Van Nguyen \& Ikeda, 2014) Used hybrid triangle mesh for fishtail rudder RANS simulations, Lutton (Lutton, 1989) specified that the 0 -mesh outperforms the $\mathrm{C}$-mesh during the evaluation of the pressure coefficients near the leading and trailing edges, on the other hand, the C-mesh demonstrates higher accuracy in predicting the wake. (Jialun, Hekkenberg, Jiang, \& Chu, 2017) indicated that the mesh type can affect the efficiency of mesh generation, the study concluded that unstructured meshes are more suitable for rudders with complex profiles, and it can predict the lift with similar accuracy to the structured mesh production.

In this paper hybrid triangle mesh and polyhedral mesh are compared, the structured mesh was studied and evaluated in many previous studies, while the application of polyhedral meshes in rudder simulation is still limited that's why it was chosen for this comparison. 


\section{Methodology}

\subsection{Geometry and boundary conditions}

NACA 0025 was chosen with a chord length $C R=1[\mathrm{~m}], \mathrm{Re}=10^{6}$, and an angel of attack $=10$ degrees, it was surrounded by two domains inner domain and outer domain, for more control over the mesh, fluid type is freshwater, the geometry and the boundary conditions are shown in the figure below:

symmetry

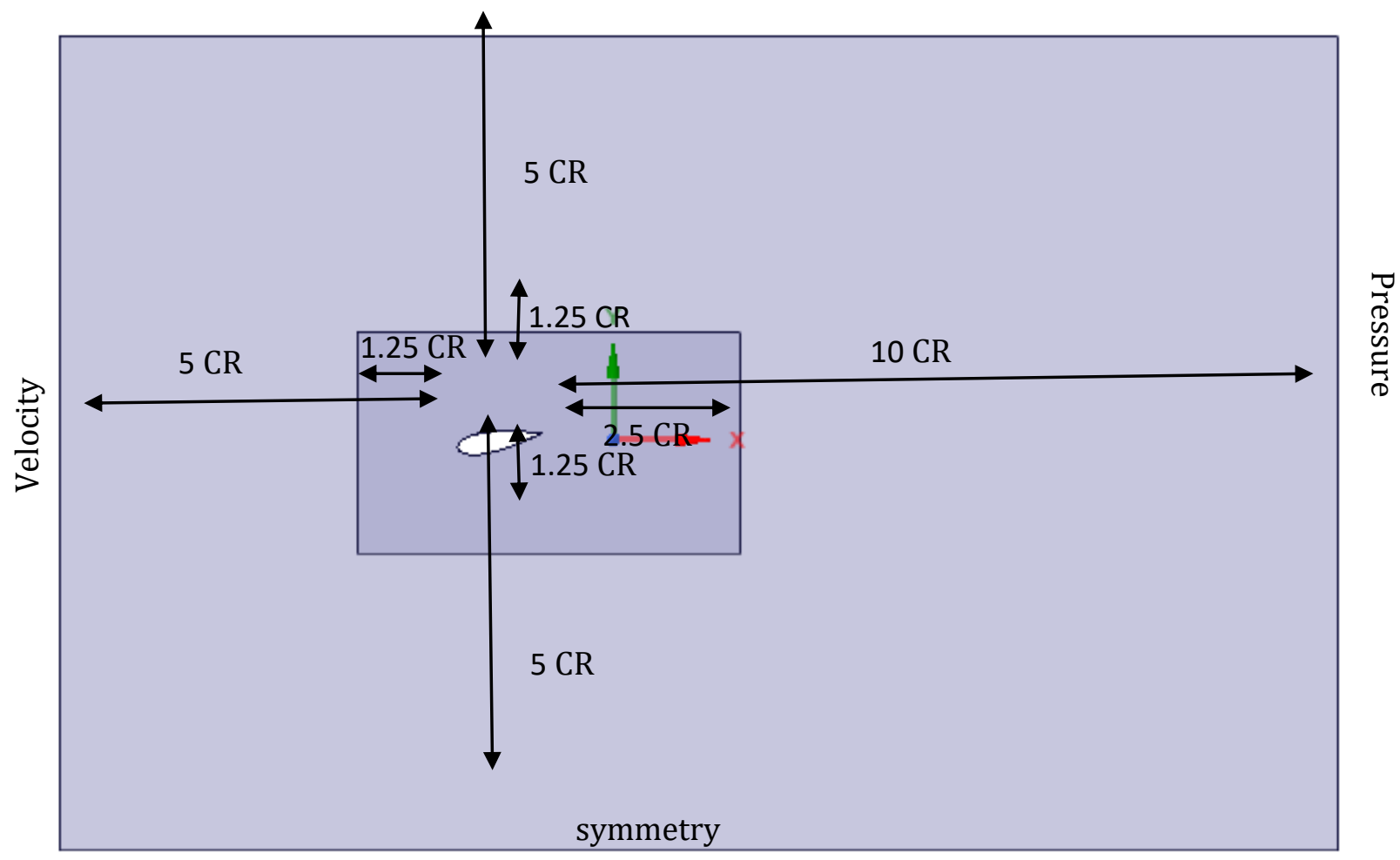

Figure 2. Geometry and boundary conditions

\subsection{Meshing}

As discussed before two types of meshes are tested, a hybrid triangle mesh and a polyhedral mesh, both meshes were created using ANSYS 2020 R1 Academic version, both meshes use a coarse, medium, and a finer mesh around the rudder, and inflation layer was also implemented, number of layers 18, growth rate 1.2. the following table shows both meshes size information:

\begin{tabular}{|l|l|l|l|}
\hline & Cells & Faces & nodes \\
\hline
\end{tabular}




\begin{tabular}{|l|l|l|l|}
\hline Hybrid triangle mesh & 450790 & 1086253 & 189881 \\
\hline Polyhedral mesh & 315614 & 775895 & 137193 \\
\hline
\end{tabular}

Table 1Mesh size information

It can be noticed that converting the mesh to a polyhedral based mesh reduced the number of the elements by $30 \%$ the mesh itself, the inner domain, and the outer domain can be seen in the following figure:

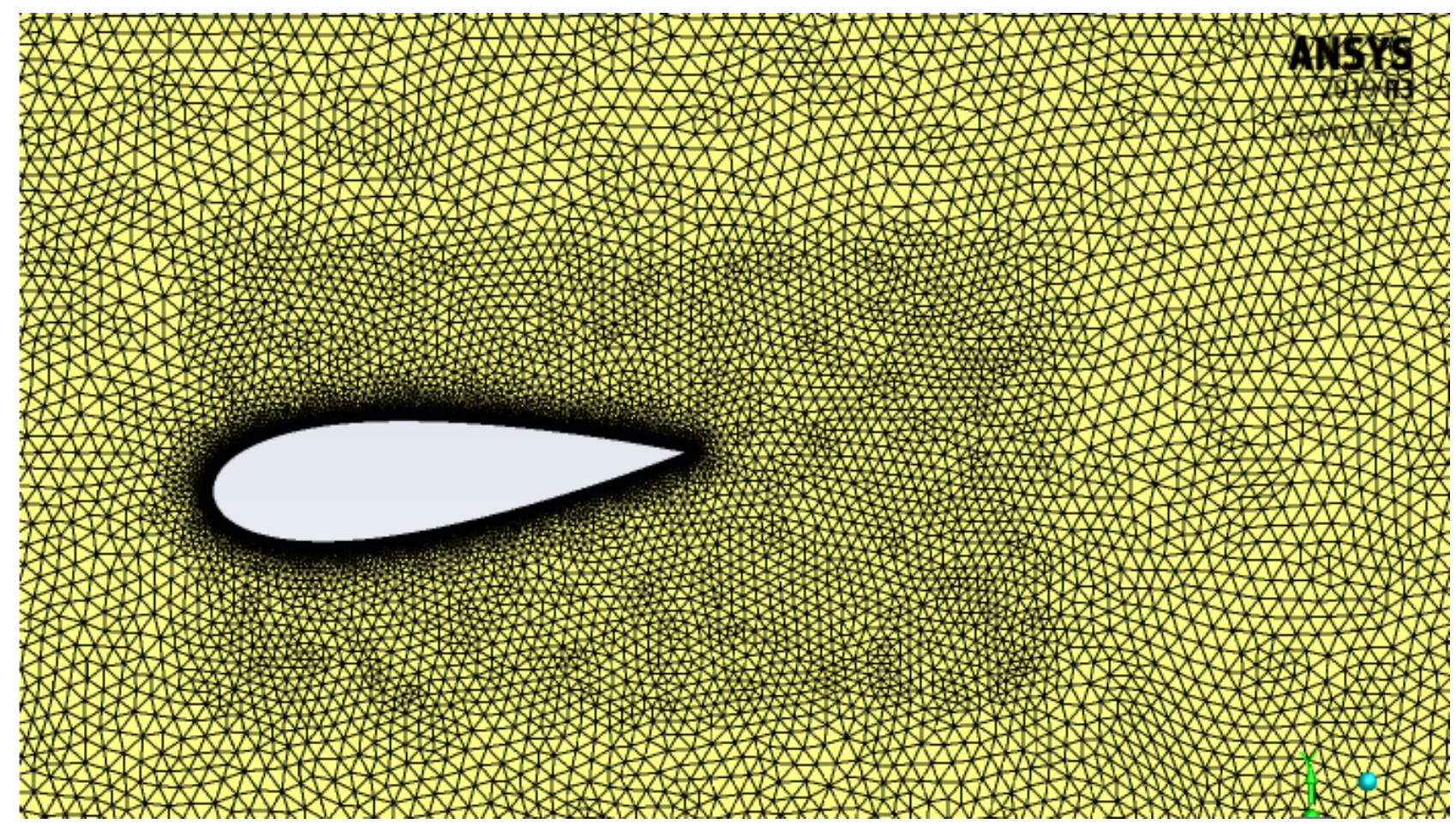

Figure 3. Mesh, outer and inner domains 


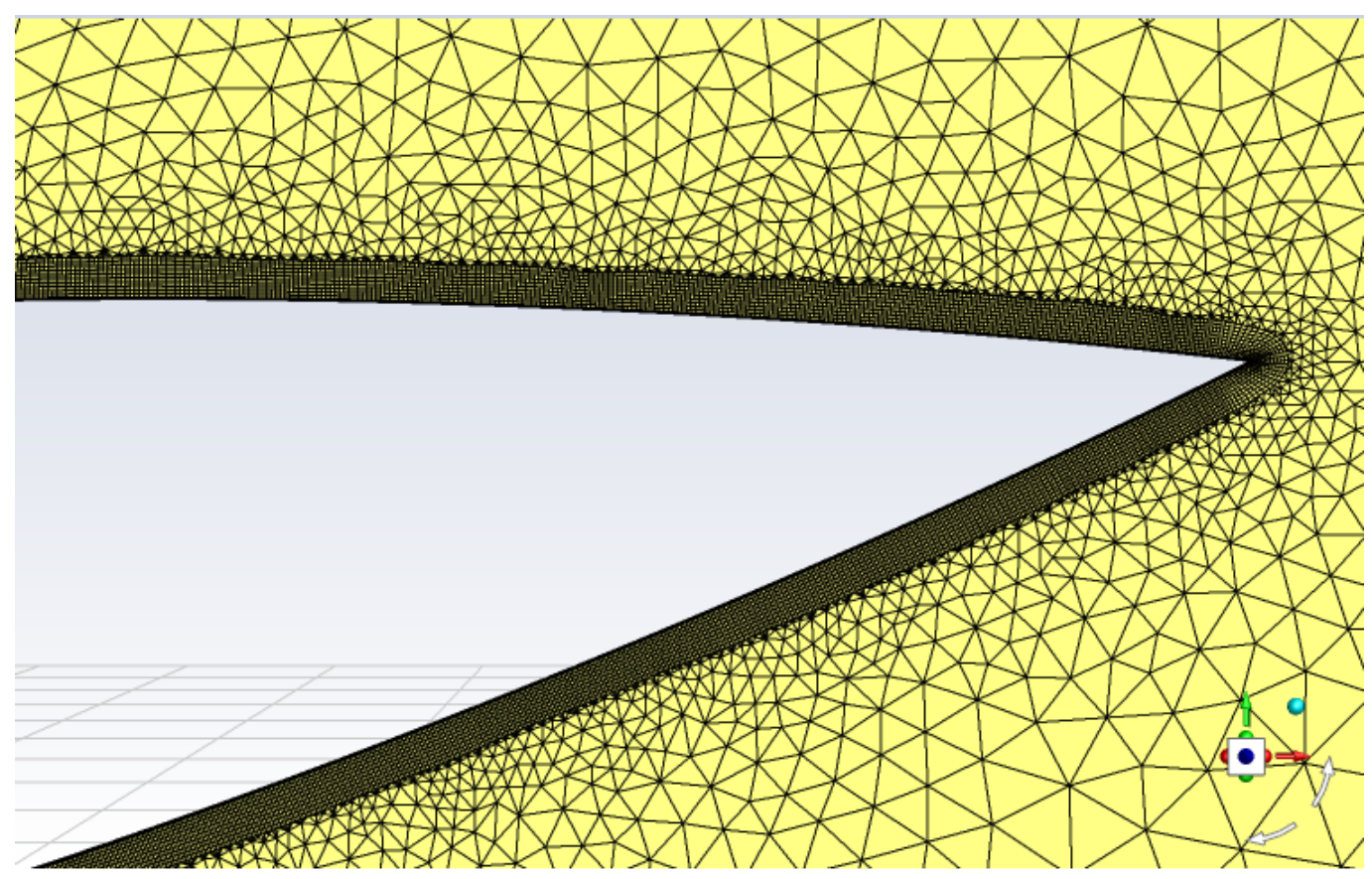

Figure 4. tetrahedral based mesh around the rudder

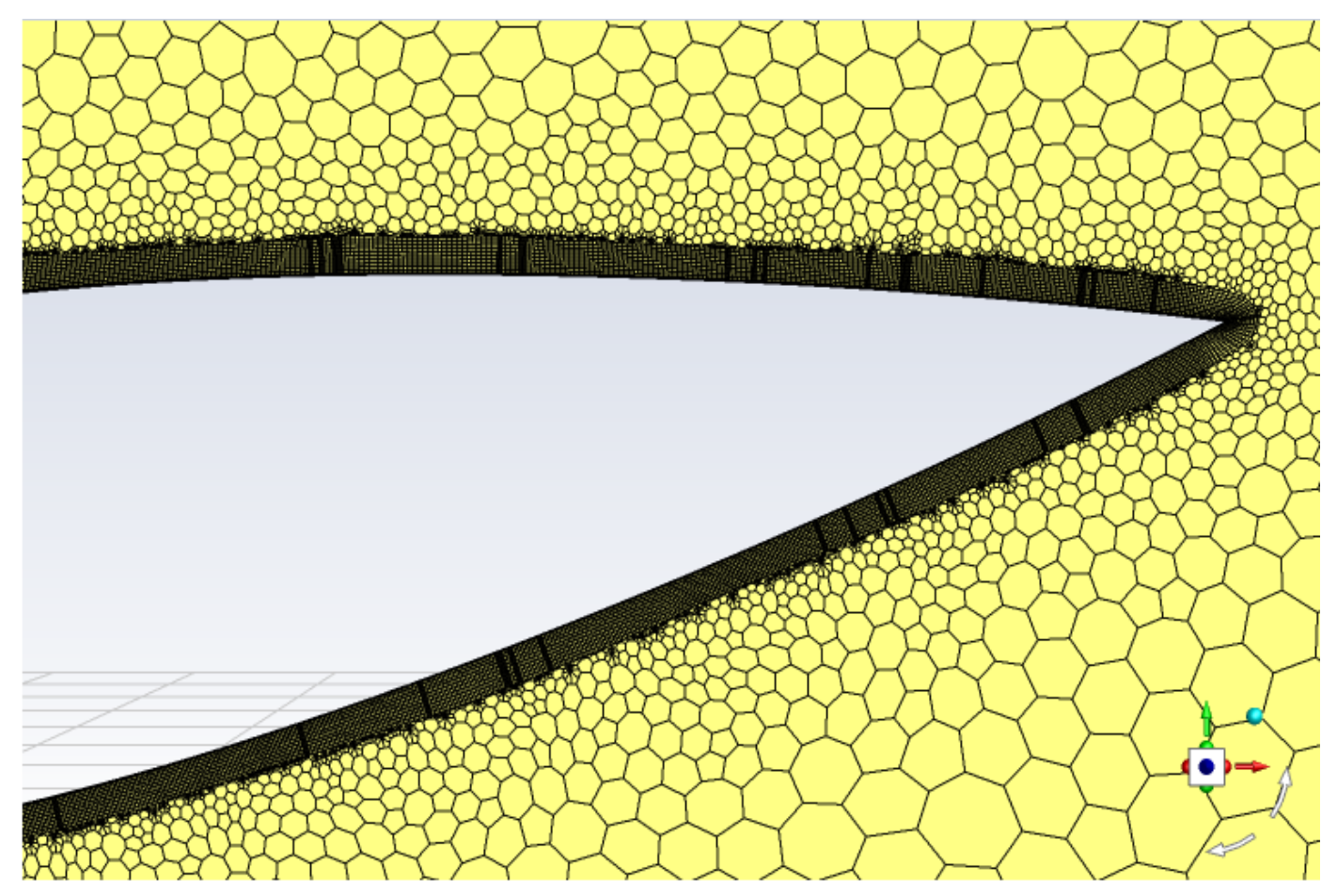

Figure 5. polyhedral mesh around the rudder

It can be seen that in both meshes the inflation layer is applied around the rudder for better near-wall treatment.

\subsection{Numerical model, SST k- $\omega$ model}


This model was created by Menter 1994, the shear stress transport model alternates between the k- $\omega$ model close to the surface and the $\mathrm{k}-\varepsilon$ model in the free-shear layers, using a blend function that can switch between these two models, this model is also capable of considering the turbulent shear stress transport, and producing the onset and the amount of flow separation under adverse pressure gradients (Yang, Gu, \& Jin, 2009). A blending F1 function is multiplied to the equations of the original Wilcox k- $\omega$ model, and the function 1-F1 is multiplied to the transformed $k-\varepsilon$ equations. Then the resulting turbulent kinetic energy $\mathrm{k}$ equation and the turbulent frequency $\omega$ equation are obtained to form the SST k- $\omega$ model:

$$
\begin{gathered}
\frac{\partial \rho k}{\partial t}+\frac{\partial}{\partial x_{j}}\left(\rho u_{i} k-\left(\mu+\frac{\mu_{t}}{\sigma_{k}}\right) \frac{\partial k}{\partial x_{j}}\right)=P_{k}-C_{\mu} \rho \omega k \\
\frac{\partial \rho \omega}{\partial t}+\frac{\partial}{\partial x_{j}}\left(\rho u_{i} \omega-\left(\mu+\frac{\mu_{t}}{\sigma_{\omega}}\right) \frac{\partial \omega}{\partial x_{j}}\right)=\alpha \frac{\omega}{k} P_{k}-\beta \rho \omega^{2}+2\left(1-F_{1}\right) \frac{\rho}{\sigma_{\omega 2} \omega} \frac{\partial k}{\partial x_{j}} \frac{\partial \omega}{\partial x_{j}}
\end{gathered}
$$

where $\rho$ is the density of the fluid, $\mathrm{k}$ and $\omega$ are the turbulent kinetic energy and its dissipation frequency, respectively and $P_{k}$ is the production of turbulent kinetic energy.

The blending function, incorporating the damped cross-diffusion derivative term in the $\omega$ equation of the SST model, using the modified definition of the turbulent viscosity for accounting for the transport of the turbulent shear stress, and the new modeling constants are all features that make this model accurate and reliable for this kind of applications. (Tahsinul, Rahman, Bin Fazle, \& Karim, 2016)

\subsection{Validation model}

Jialun Liu and Robert Hekkenberg (Liu \& Hekkenberg, Hydrodynamic Characteristics of Twin-Rudders at Small Attack Angles, 2015) did research on hydrodynamic characteristics of different types of rudders including the NACA 0025 used in this paper, the authors validated their results by comparing it with the computed results by CFL3D (NASA LaRC, USA), FUN3D (NASA LaRC, USA), and NTS (NTS, Russia) from the Langley Research Centre (2014). And calculated the relative errors, and they found that for unstructured meshes the model showed very good accuracy calculating the lift coefficient CL as the relative error varied for different angles of attack between [-1.33] degrees and [-2.17] degrees. while for the drag coefficient CD the accuracy was not as good with a relative error between [2.47] and [20.07] and the error was increasing directly with the increase of the angle of attack, the results showed that the model tends to under-estimate the lift coefficient and overestimate the drag coefficient, and they returned the underestimation of the lift coefficient to the numerical diffusion while the overestimation of the drag coefficient was returned to the assumption of fully turbulent flow. The results were considered sufficient as the lift coefficient determines the rudder effectiveness.

The details of their model can be seen in the following table:

\begin{tabular}{|l|l}
\hline Property & Setting \\
\hline
\end{tabular}




\begin{tabular}{|l|l|}
\hline Reynolds number & 6 million \\
\hline Solver & Pressure-Based Steady \\
\hline Materials & Water liquid \\
\hline Viscous Model & k- $\omega$ SST \\
\hline Pressure-Velocity Coupling & Coupled \\
\hline Gradient & Least Squares Cell-Based \\
\hline Pressure & Second Order \\
\hline Momentum & Second-Order Upwind \\
\hline Turbulent Kinetic Energy & Second-Order Upwind \\
\hline Specific Dissipation Rate & Seconder Upwind \\
\hline Convergence Criteria & \\
\hline
\end{tabular}

Table 2. Model settings for CFD calculations

For the NACA 0025 rudder at an angle of attack of 10 degrees the model predicted the following values: $\mathrm{CL}=0.919, \mathrm{CD}=0.018$

\section{Results}

This study compared two types of meshing approaches both done by the ANSYS meshing tool the tetrahedral elements were converted into polyhedral elements using the FLUENT converting tool, the simulation setup from the validation model was maintained including the Reynolds number of $6.10^{6}$, standard wall function was used, Y plus values were kept below 300 except for some points which is understandable considering the high Reynolds number. Y plus can be calculated from the following formula:

$$
y^{+}=\frac{u_{*} y}{\vartheta}(3)
$$


Where is $u_{*}$ is the friction velocity at the nearest wall, $y[\mathrm{~m}]$ is the distance to the nearest wall, and $\vartheta$ [m $\mathrm{s}^{-1}$ ] is the local kinematic viscosity of the fluid. (ANSYS, 2018)

\subsection{Convergence Analysis:}

Figure 5 and figure 6 show the convergence plots of both mesh types, A predefined standard convergence criterion of $10^{-5}$ was applied. Convergence analysis in CFD depends on two factors, the first is the mesh size and discretization scheme, and the second is the complexity of the geometry, and since the same geometry is used for both models, then the difference is related to the mesh itself.

Figure 5 shows that the tetrahedral-based mesh model converged at a much higher number of iterations (1100-1200) iteration, while the polyhedral mesh-based model converged in a much smaller number of iterations (200-225) iteration. It can be seen clearly that polyhedral elements converged at a lower number of iterations and the fact that converging tetrahedral elements to polyhedral elements reduced the number of elements (as it can be seen from table 1) should be considered also, but as a final result of using polyhedral elements in rudder simulation applications it can be stated that solution converges faster which leads to savings in time and computational resources of about $80 \%$. While

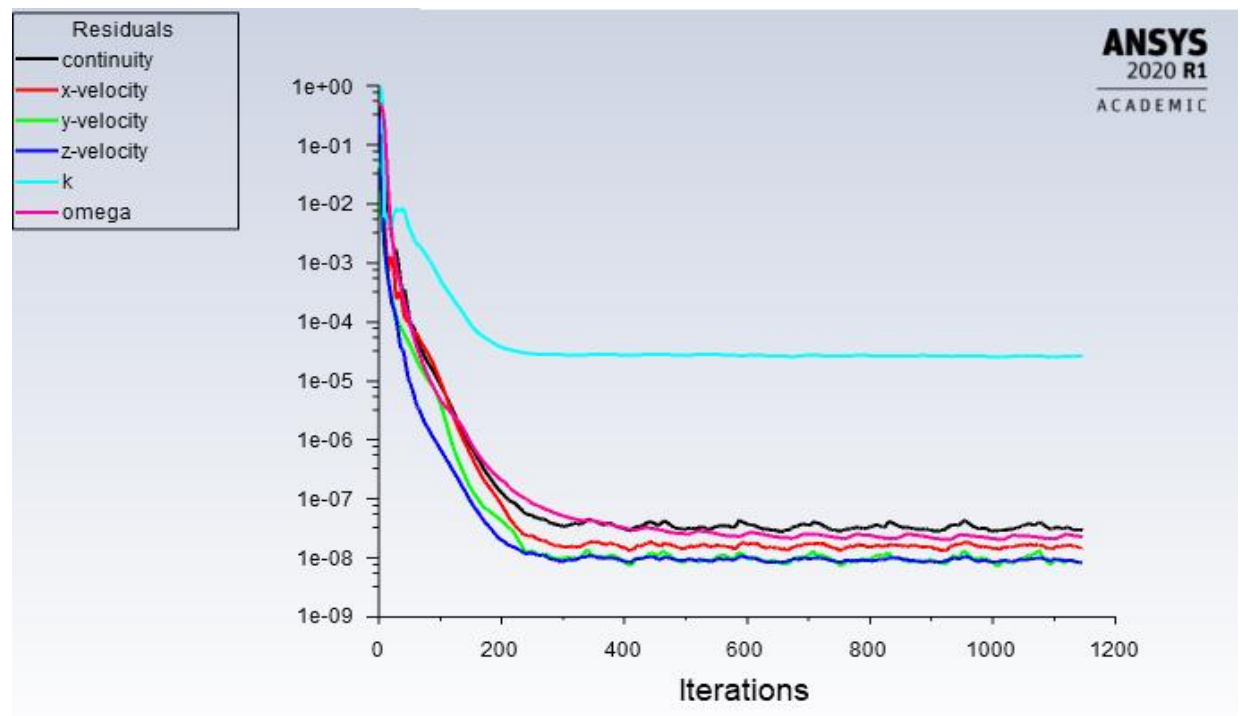

Figure 6. Convergence plot for the tetrahedral mesh model 


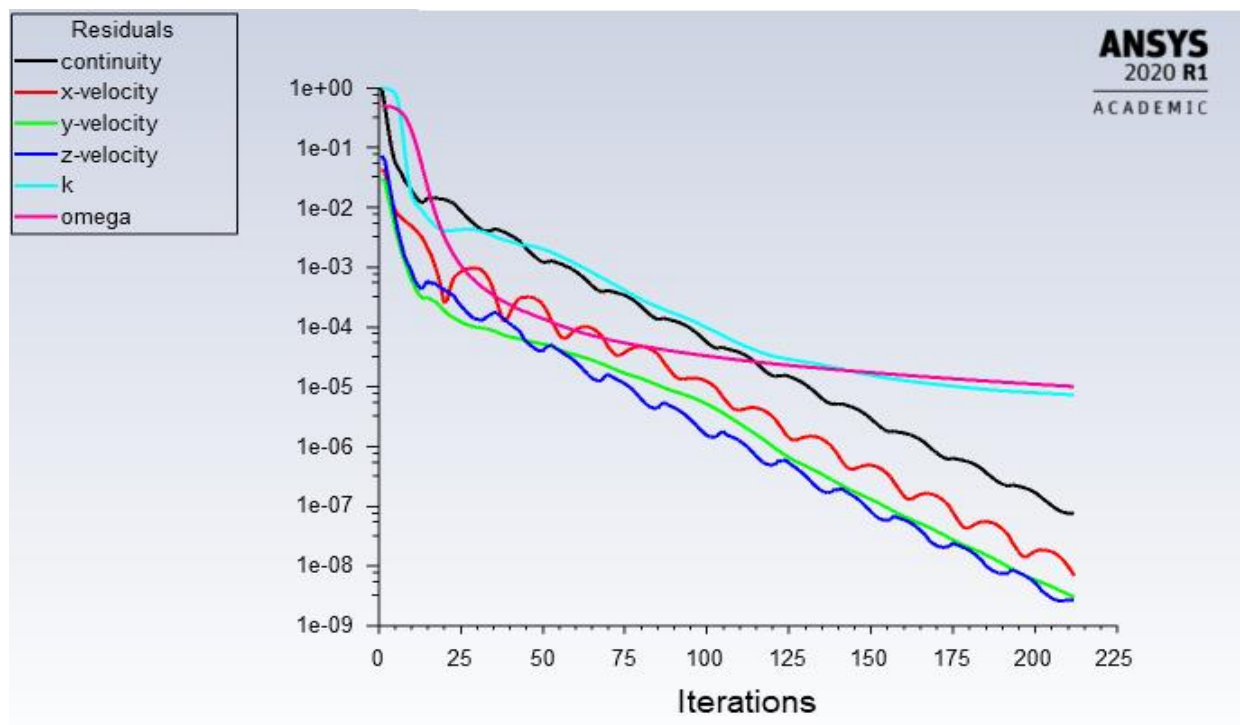

Figure 7. Convergence plot for the polyhedral mesh model

For the overall calculation time, calculations based on the polyhedral meshes were almost three times faster than calculations based on tetrahedral meshes.

\subsection{Lift and drag coefficients}

Figures (8)(9) shows that both lift and drag coefficients started to converge after 100 iterations and fully converged after between (200-225) iterations, while figures (10)(11) shows that both coefficients in the tetrahedral mesh-based model started to converge after 200 iterations and fully converged only after between (1100-1200) iterations.

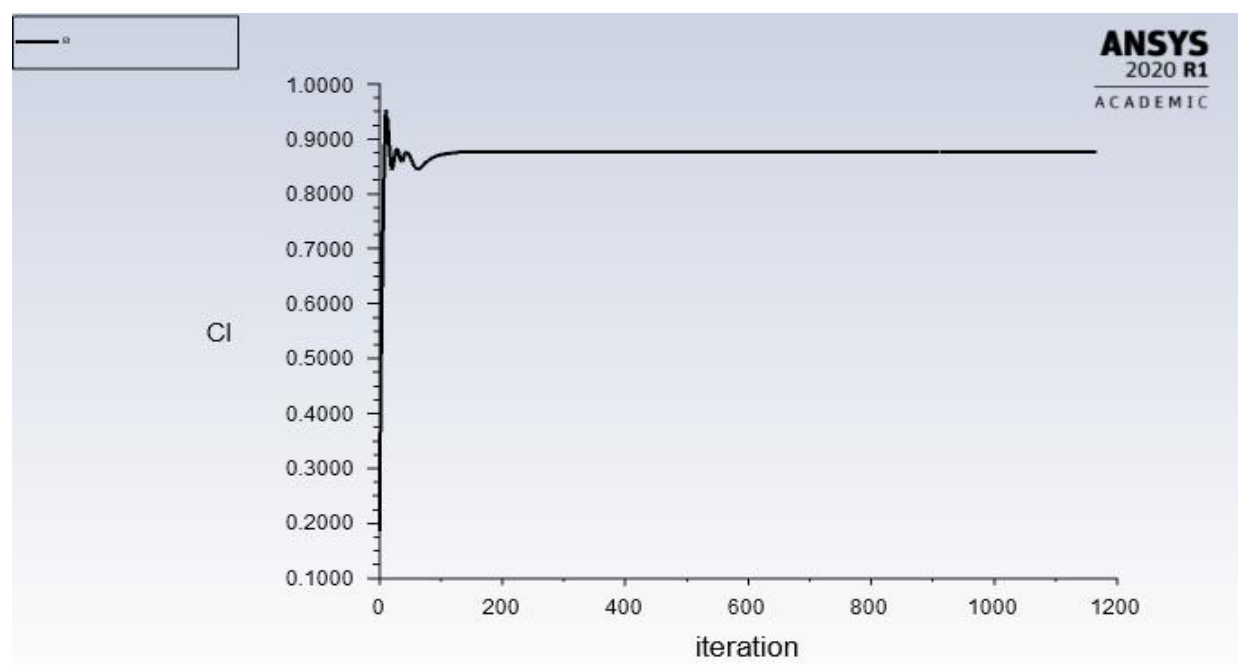

Figure 8. Lift coefficient convergence for the tetrahedral based model. 
International Journal of Engineering and Management Sciences (IJEMS) Vol. 6. (2021). No. 2

DOI: 10.21791/IJEMS.2021.2.21.

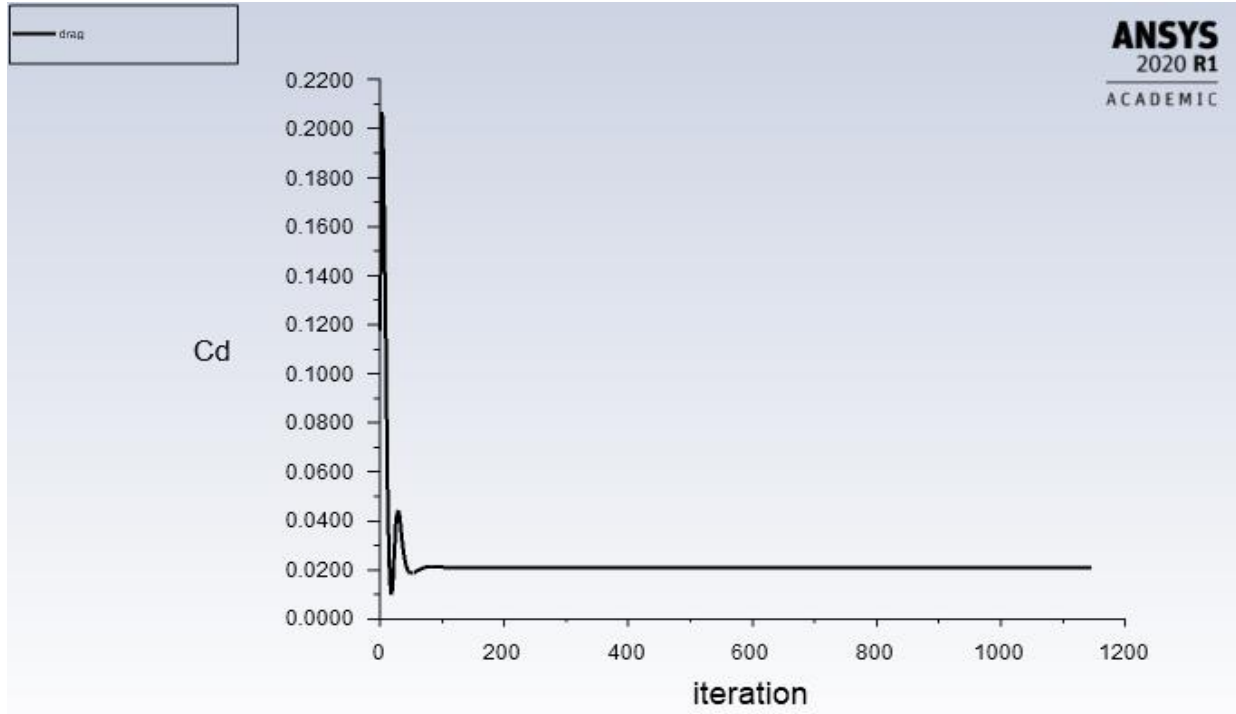

Figure 9. Drag coefficient convergence for the tetrahedral based model.

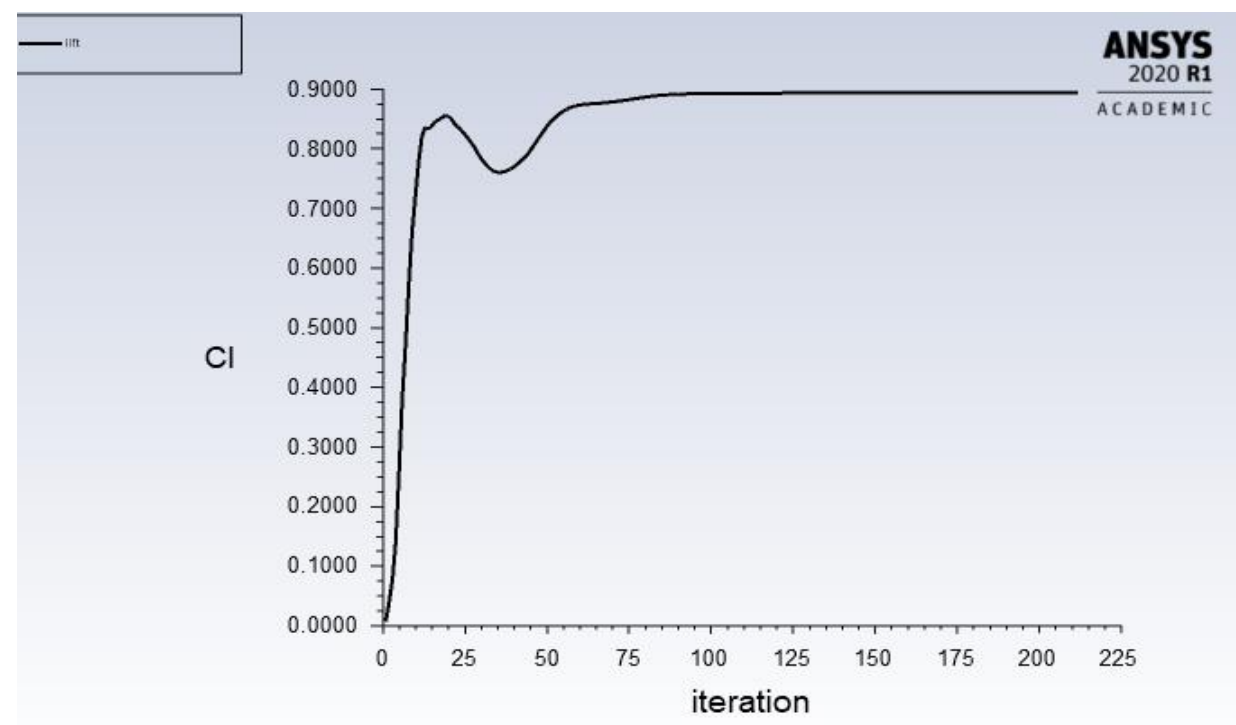

Figure 10. Lift coefficient convergence for the polyhedral-based model. 


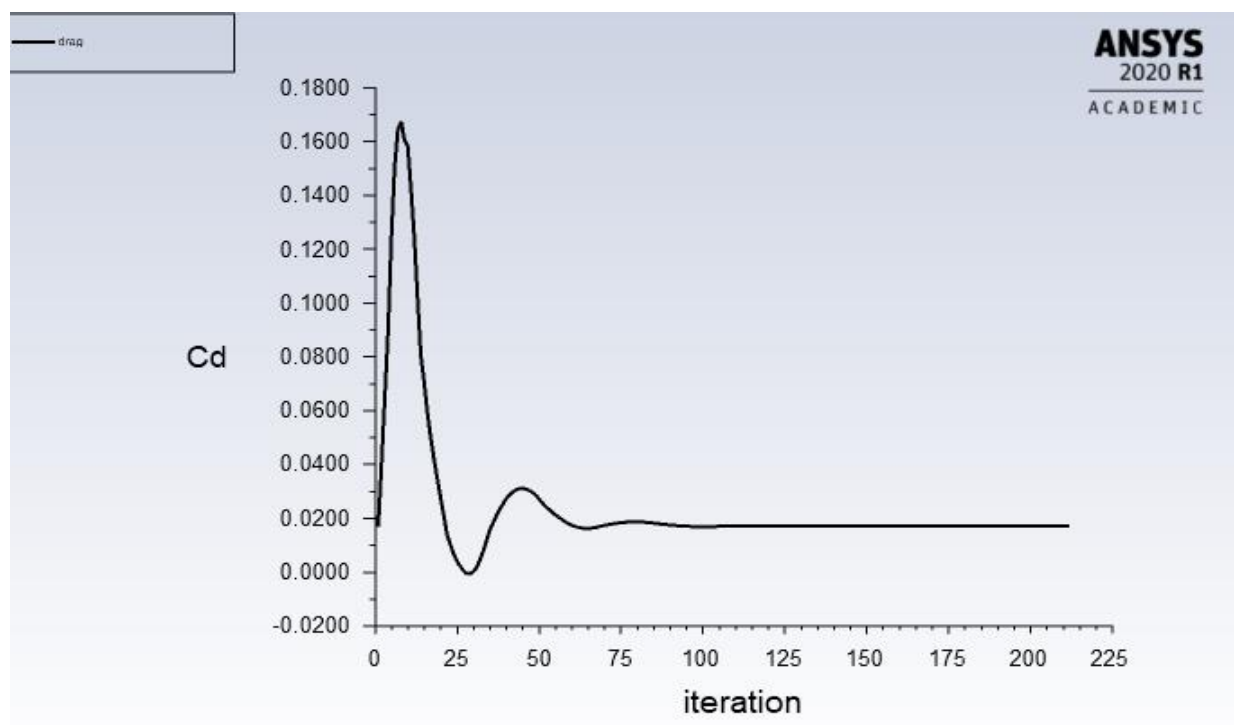

Figure 11. Drag coefficient convergence for the polyhedral-based model.

The results of lift and drag coefficients at an angle of attack of 10 degrees presented in table 3 show that the polyhedral mesh-based model underestimated both the lift and the drag coefficient compared to the validation model, while the tetrahedral-based mesh model underestimated the lift coefficient and overestimated drag coefficient.

\begin{tabular}{|l|l|l|}
\hline & Lift coefficient & Drag coefficient \\
\hline Polyhedral mesh & 0.897 & 0.017 \\
\hline Hybrid triangle mesh & 0.876 & 0.020 \\
\hline
\end{tabular}

Table 3. Lift and drag coefficients values.

Calculating the relative and absolute error for both coefficients compared to the validation model using the following equation:

$$
\begin{aligned}
& \text { absolute error }=\mid \text { validation value }- \text { predicted value } \mid(4) \\
& \text { relative error }=\mid \text { absolute error } / \text { validation value } \mid(5)
\end{aligned}
$$

Gave the following results:

\begin{tabular}{|l|c|c|c|c|}
\hline \multirow{2}{*}{} & \multicolumn{2}{|c|}{$\Delta C L$} & \multicolumn{2}{c|}{$\Delta C D$} \\
\cline { 2 - 5 } & Abs error & relative error & Abs error & relative error \\
\hline Polyhedral mesh & 0.022 & $2.394 \%$ & 0.001 & $5.56 \%$ \\
\hline Hybrid triangle mesh & 0.043 & $4.68 \%$ & 0.003 & $15 \%$ \\
\hline
\end{tabular}

Table 4. Relative error

It can be clearly stated that the polyhedral based mesh model gave higher accuracy in predicting the lift and drag coefficients in a smaller number of iterations which means that it also needed less time and less computational resources, and this can be caused by the previously mentioned advantages of polyhedral elements like the larger number of neighboring elements. And the more efficient exchange 
of mass over a larger number of faces, which reduces the numerical diffusion effects caused by flows that are not perpendicular to any of the cell faces.

\subsection{Pressure}

Both mesh models captured the static pressure distribution around the rudder the flow is trapped behind the surface of the rudder which creates two high-pressure tips one of them and the most obvious one is at the leading edge of the rudder, while the second one can be seen at the trailing edge of the rudder.

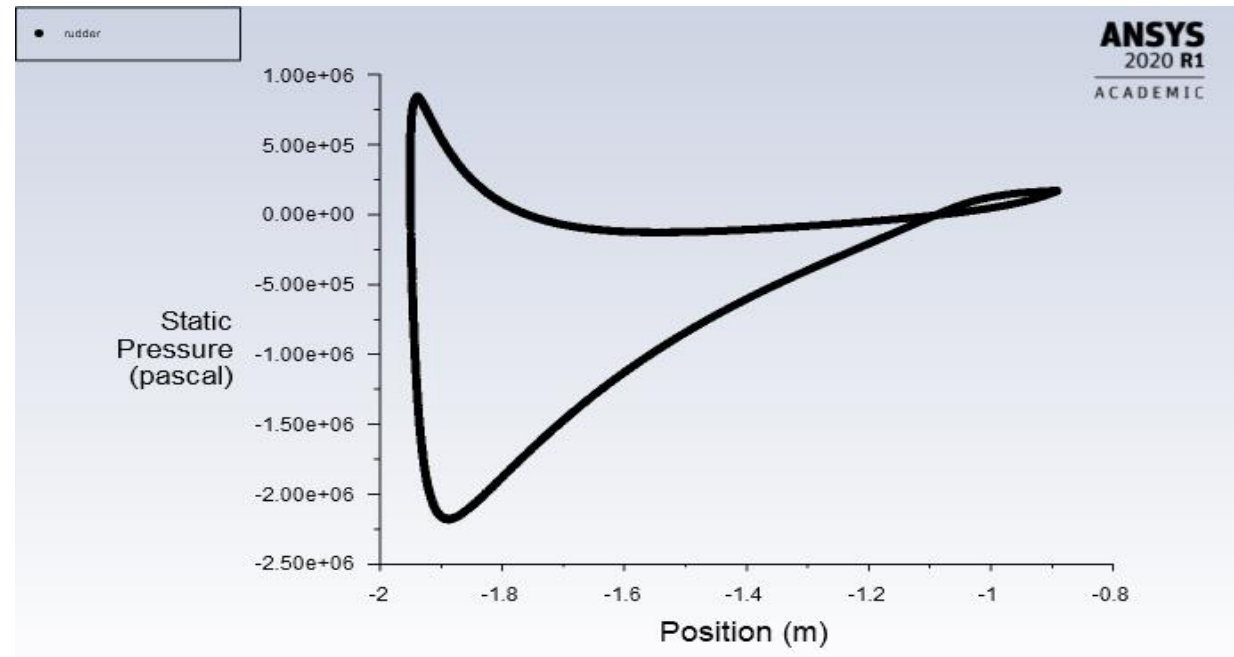

Figure 12. static pressure of the tetrahedral mesh-based model

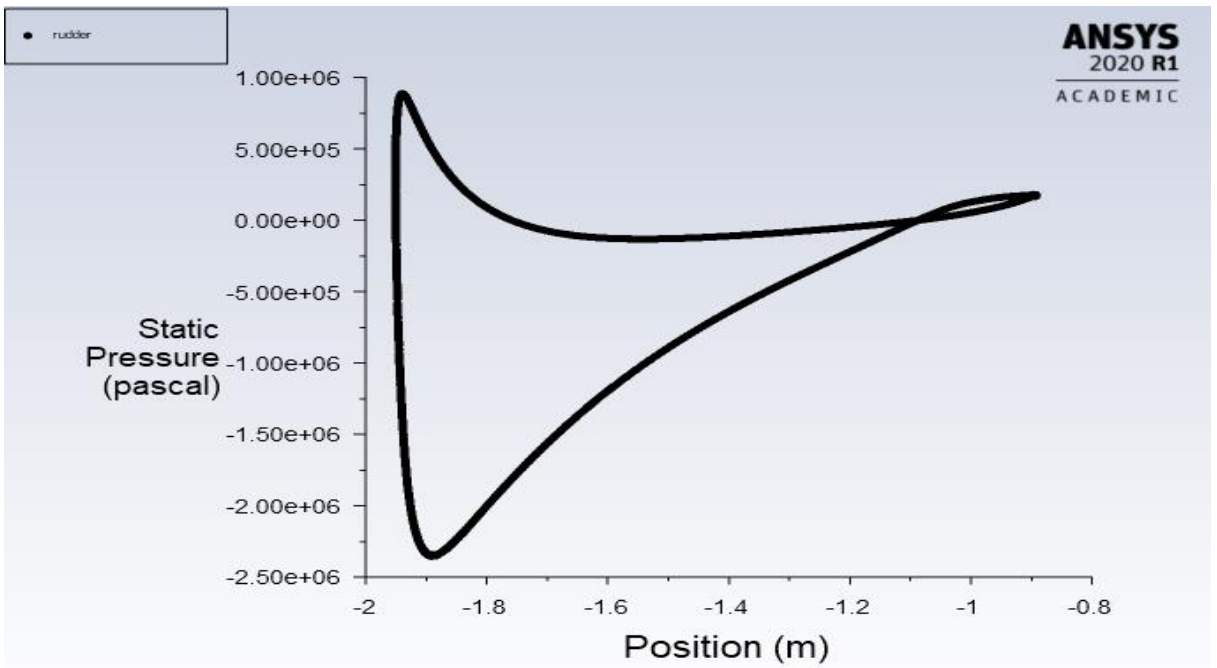

Figure 13. static pressure of the polyhedral mesh-based model

\subsection{Turbulent kinetic energy}

Figures 11 and 12 show that both models were able to predict similar general characteristics of the turbulent kinetic energy, but the numerical diffusion is more obviously presented in the tetrahedral based system due to the difficulties in the mass exchange between tetrahedral element faces, while 
polyhedral elements could overcome this problem thanks to its multi-surface shape which offers more surfaces perpendicular to the flow direction.

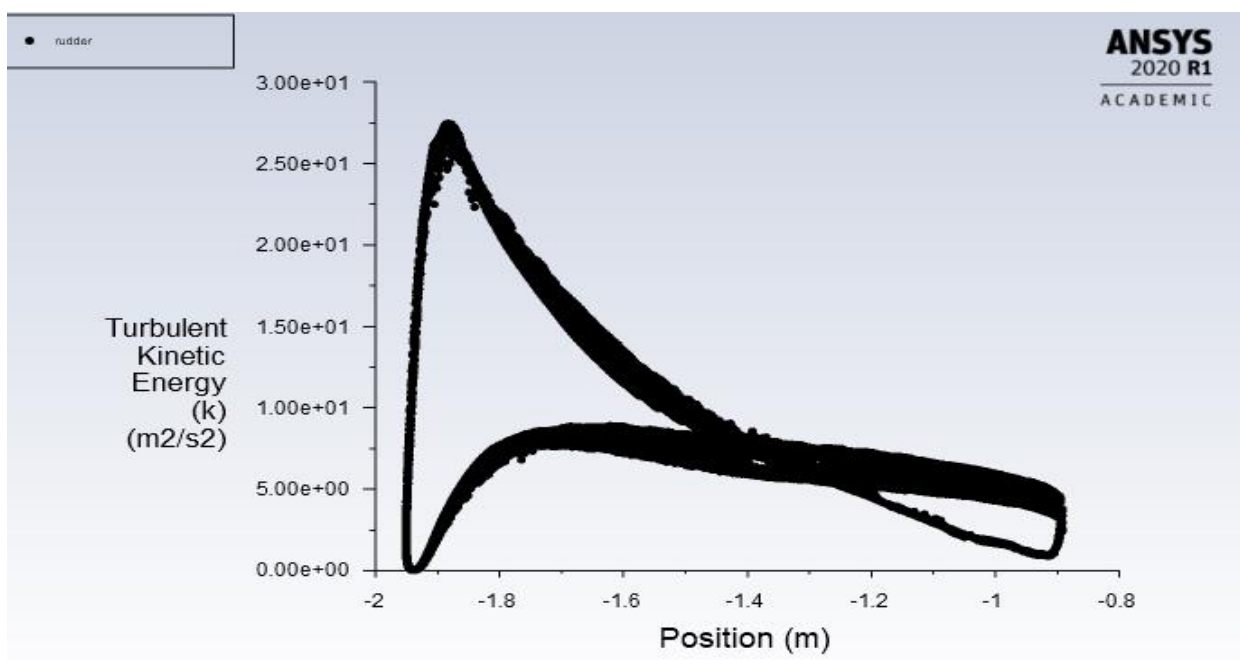

Figure 14. Turbulent kinetic energy for the tetrahedral based mesh model

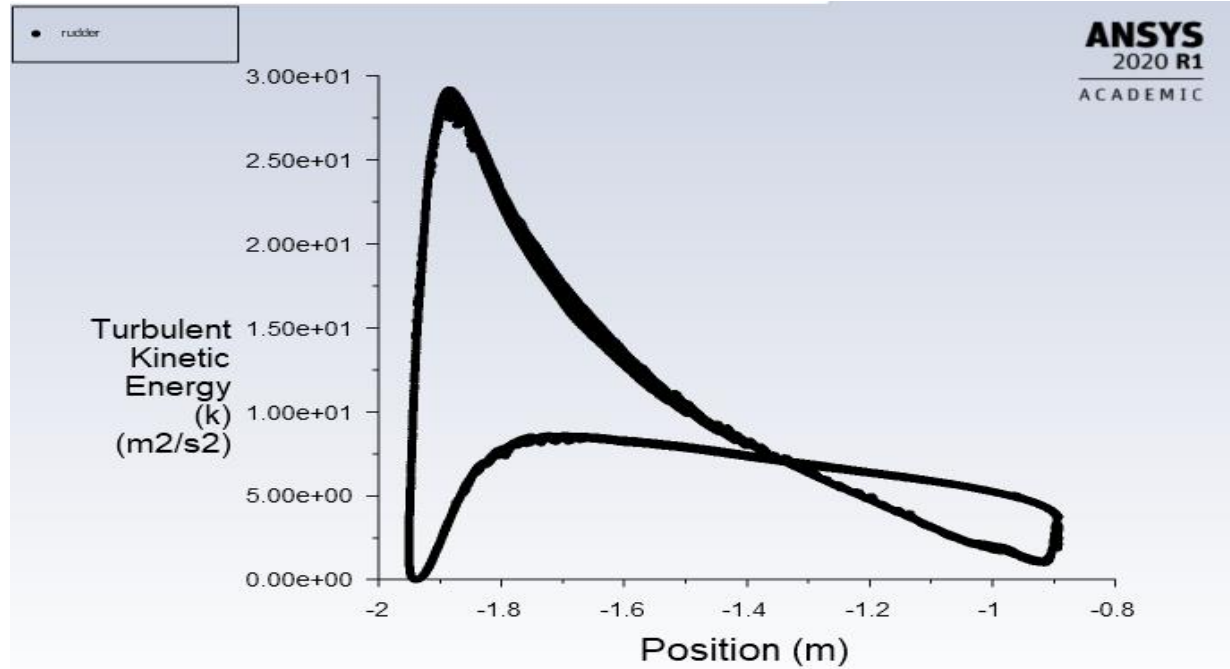

Figure 15. Turbulent kinetic energy for the polyhedral based mesh model

Both meshes were able to predict similar velocity fields around the rudder profile.

\subsection{Mesh quality}

Mesh quality is one of the most critical factors when it comes to evaluating the stability and accuracy of the obtained results, mesh quality can be judged depending on different criteria, like the aspect ratio and the orthogonal quality, in this case, study the orthogonal quality index was used to evaluate the polyhedral and tetrahedral elements, as it is an essential parameter in almost all CFD based tools (CANONSBURG, 2012). Orthogonal quality ranges between 0 and 1 , the closer to 0 value the worse the quality, and the closer to 1 the more optimal quality. 


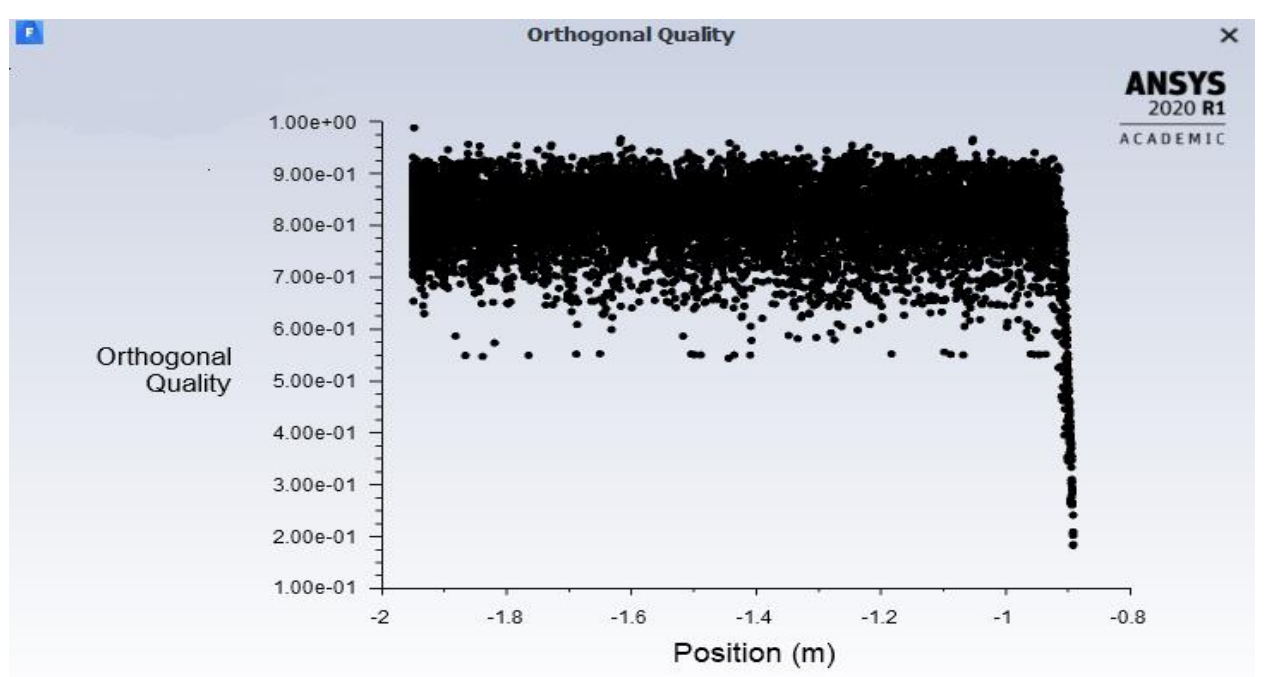

Figure 16. orthogonal quality of the tetrahedral based mesh

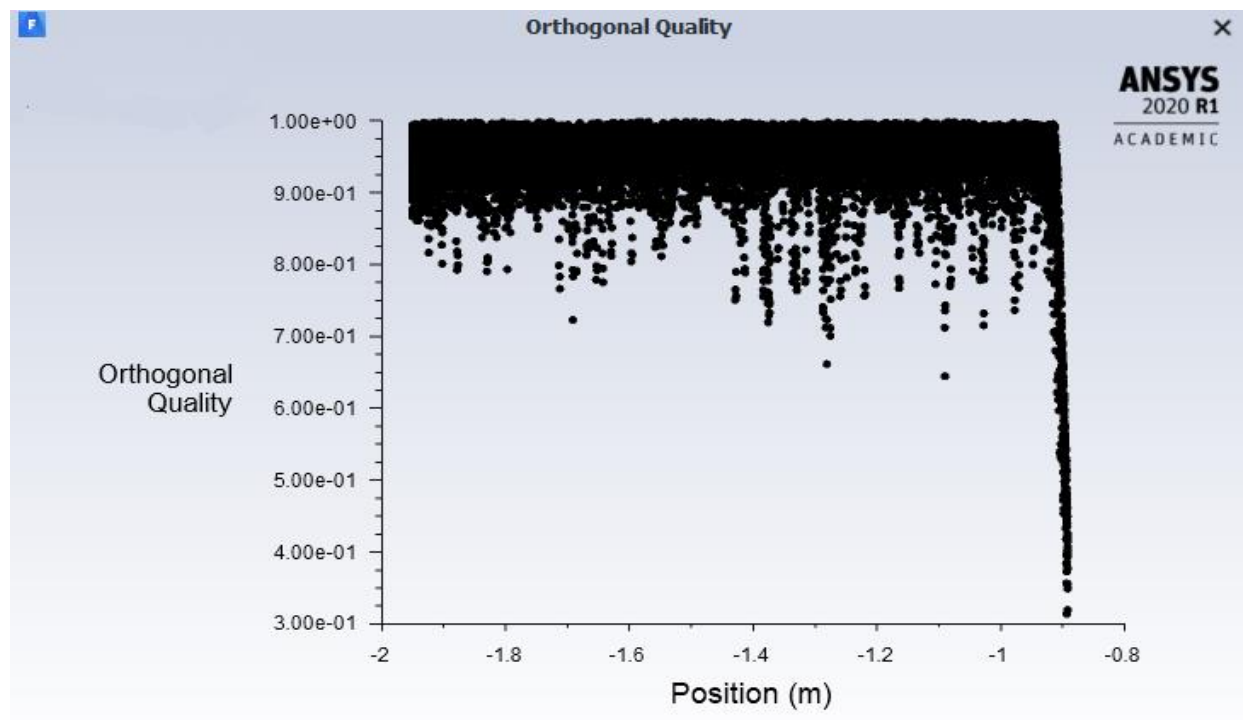

Figure 17. orthogonal quality of the polyhedral based mesh

The two previous figures indicate that most of the polyhedral mesh elements have an orthogonal quality between 0.85 and 1 while for the tetrahedral-based mesh most of the elements have orthogonal quality between 0.7 and 0.95 , which refers to a more optimal quality in favor of the polyhedral based mesh.

\section{Conclusion}

This study concentrated at the efficiency of the polyhedral elements in solving the flow around ship rudder problems, and it compared the polyhedral elements to the tetrahedral elements, the result were validated by comparison with an already validated CFD model, the lift and drag coefficient were calculated and compared, the pressure around the rudder also was studied in addition to the turbulent kinetic energy and the velocity, the convergence was analyzed, polyhedral elements proved to be more efficient, it needed less iteration to fully converge and consumed less time and less computational resources, it was also able to overcome the numerical diffusion problem witnessed in tetrahedral 
elements, even comparing mesh quality according to the orthogonal quality criteria showed that polyhedral elements have better mesh quality, more studies should be concluded to find optimal cases of tetrahedral based meshes to lead to a better converging to polyhedral, further studies can be made also to evaluate the efficiency of the newer mosaic mesh supported and introduced lately by ANSYS as the core element is the polyhedron.

\section{References}

[1] ANSYS, I. (2018). Ansys.com. Retrieved from www.simutechgroup.com: https://www.simutechgroup.com/images/easyblog_articles/7/ANSYS-Fluent-MosaicTechnology-Automatically-Combines-Disparate-Meshes-with-Polyhedral-Elements-for-FastAccurate-Flow-Resolution---White-Paper.pdf

[2] CANONSBURG, T. (2012). ANSYS FLUENT User Guide. ANSYS INC.

[3] Jialun, L., Hekkenberg, R., Jiang, Z., \& Chu, X. (2017). Mesh Properties for RANS Simulations of Aerofoil-Shape Rudder Hydrodynamics. 10th International Workshop on Ship and Marine Hydrodynamics. Keelung.

[4] Kim, S. (2011). CFD as a seakeeping tool for ship design. International Journal of Naval Architecture and Ocean Engineering.

[5] Liu, J., \& Hekkenberg, R. (2015). Hydrodynamic Characteristics of Twin-Rudders at Small Attack Angles. 12th International Marine Design Conference (pp. 177-188). Delft,Netherlands: Delft University of Technology.

[6] Liu, J., \& Hekkenberg, R. (2016). 3D RANS simulations of shallow water effects on rudder hydrodynamic characteristics. Proceedings 2016 International Conference on Maritime Technology (pp. 35-39). Harbin: Harbin Engineering University.

[7] Lutton, M. (1989). Comparison of C-and O-grid Generation Methods Using a NACA 0012 Airfoil. Master thesis. Air Force Institute of Technology.

[8] Michalcová, V., \& Kotrasova, K. (2020). The Numerical Diffusion Effect on the CFD Simulation Accuracy of Velocity and Temperature Field for the Application of Sustainable Architecture Methodology. Sustainability.

[9] Sadrehaghighi, I. (2020). Mesh Generation in CFD. AN N A P O L I S.

[10] Shepherd, J., \& Johnson, C. (2008). Hexahedral mesh generation constraints. Springer.

[11] Sosnowski, M., Krzywanski, J., \& Gnatowska, R. (2016). Polyhedral meshing as an innovative approach to computational domain discretization of a cyclone in a fluidized bed CLC unit. Energy and Fuels . Czestochowa: EDP Sciences.

[12] Sosnowski, M., Krzywanski, J., \& Gnatowska, R. (2017). Polyhedral meshing as an innovative approach to computational domain discretization of a cyclone in a fluidized bed CLC unit . E3S Web of Conferences.

[13] Tahsinul , T. H., Rahman, M., Bin Fazle, A., \& Karim, M. (2016). Numerical Prediction of Flow Past a Marine Rudder. 10th International Conferenceon Marine Technology, (pp. 59-66). MARTEC . 
[14] Van Nguyen, T., \& Ikeda, Y. (2014). Development of fishtail rudder sections with higher maximum lift coefficients. 24th International Ocean and Polar Engineering Conference, (pp. 940947). Busan.

[15] Whitworth, S. (2007). Rudder Design Investigation: CFD. Lloyd's Register Technical.

[16] Yang, Y., Gu, M., \& Jin, X. (2009). NEW INFLOW BOUNDARY CONDITIONS FOR MODELING THENEUTRAL EQUILIBRIUM ATMOSPHERIC BOUNDARY LAYER IN SST k- $\omega$ MODEL. The Seventh Asia-Pacific Conference on Wind Engineering. Taipei, Taiwan. 